lighter atoms does not depend very much on the size of the molecule, or the number of these lighter atoms, provided that (as is generally the case for organic molecular structures) there is no definite integral relationship between such intra-molecular periodicities as may exist and the cell dimensions of the crystal. As the molecule gets larger, the geometrical structure factors on the average depart further from their possible maxima. The contributions from the various atoms tend to cancel each other out, and we are left with a small residual. This is, in fact, one of the essential difficulties in analysing a complex structure by trial. A comparatively small change in the structure may mean a big change in these small residuals. But the contribution from the heavy atom is constant and will continue to dominate the situation. In a recent example ${ }^{6}$, a nickel atom, representing only about 10 per cent of the total molecular weight, was found to determine practically all the phase constants.

It may be going too far to suggest that the insulin structure could be determined in this way. The molecule does, however, contain a few zine atoms, and if these could be replaced by mercury, as has been suggested, a very profitable study might ensue. Of course there are formidable difficulties. If the reflections are swamped in the manner described, much greater accuracy is required in the intensity determinations to reveal the small differences. In the same way, a higher numerical accuracy is required in the calculations to reveal the minor maxima and differentiate them from spurious effects. Recent results have shown that these difficulties can be overcome in a moderately complex molecular structure?

Davy Faraday Laboratory, J. Monteath Robertson.

Royal Institution, London, W.I.

Dec. 28.

${ }^{1}$ Langmuir and Wrinch, NATURE, 142, 581 (1938); Wrinch, NatURE, 142, 955 (1938); Neville, NATURE, 142, 994 (1938).

${ }^{2}$ Patterson, Z. Krist., 90, 517 (1935).

${ }^{3}$ Crowfoot, Proc. Roy. Soc., A, 164, 580 (1938).

- Harker, J. Chem. Phys., 4, 381 (1936); Llewellyn, Cox and Goodwin, J. Chem. Soc., 883 (1937).

- Robertson, J. Chem. Soc., 615 (1935) ; 1195 (1936).

- Robertson and Woodward, J. Chem. Soc., 219 (1937).

${ }^{7}$ Robertson and Woodward, $J$. Chem. Soc., (1939, in preparation).

\title{
A Temperature Effect in Laue Photographs
}

DURING a study of age-hardening in single crystals of aluminium containing 4 per cent copper, certain anomalies in the Laue photographs were noticed ${ }^{1}$.

[100]

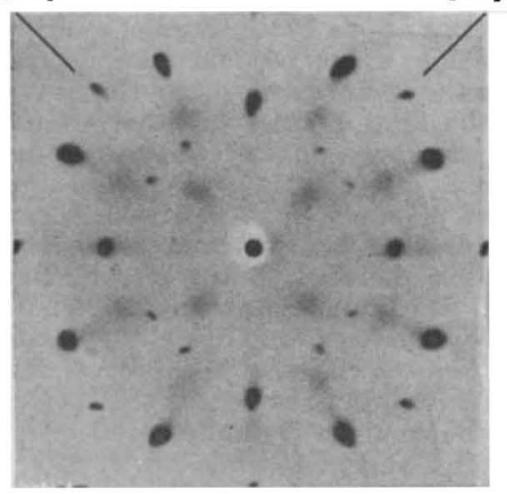

Aluminium at $20^{\circ} \mathrm{C}$.

[010]

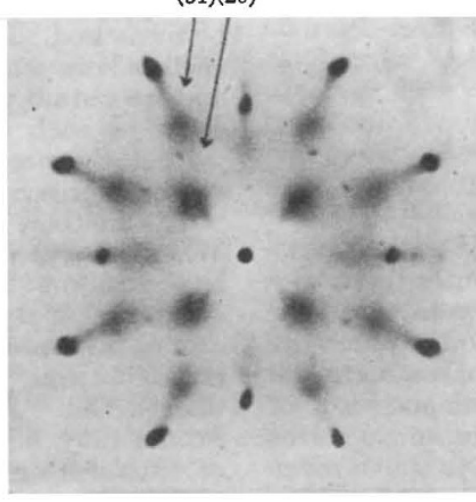

Aluminiumat $550^{\circ} \mathrm{C}$.
Some of these were associated with the progress of hardening, but others appeared to be due to another cause. Further investigation showed the presence on the Laue photographs of single crystals of aluminium, of certain faint streaks also observed in photographs of the alloy. When photographed at $550^{\circ} \mathrm{C}$., the intensity of these streaks or ill-defined spots greatly increased. Similar effects were observed in sodium chloride and magnesium oxide. The effect observed in sodium chloride at room temperature has already been noted by Wadlund ${ }^{2}$, and an explanation of its origin given by Zachariasen ${ }^{3}$. The fact that the effect becomes much more intense when the temperature is raised suggests that it is due to thermal vibrations of the lattice. In diamond at $500^{\circ} \mathrm{C}$. a faint effect similar to that obtained with the other crystals is present, although little is to be seen at room temperature.

The photographs reproduced herewith illustrate the effect in aluminium. The X-ray beam is parallel to $(001)$ and $(110)$ is vertical ; they were taken with a silver target with $60 \mathrm{kv}$. peak on the tube. The intense spots near the centre are largely due to the silver characteristic radiation, and have also been recorded with monochromatic radiation yielding a cross grating' pattern in which the spots have indexes (20) and (31).

\section{G. D. Preston.}

Metallurgy Department,

National Physical Laboratory,

Teddington, Middlesex.

Dec. 8.

1 Nature, 142, 569 (1938).

2 Phys. Rev., 53, 843 (1938)

${ }^{3}$ Phys. Rev., 53, 844 (1938).

\section{Flavo-Protein of the Heart Muscle Tissue}

I HAVE shown previously ${ }^{1}$ that the coenzyme of the $d$-amino-acid oxidase is present not only in liver and kidney tissues, which exhibit the $d$-amino acid oxidase activity, but also in skeletal and heart muscles devoid of this enzyme. If washed muscle tissue is added to the alanine test (coenzyme-free $d$-amino acid oxidase and alanine), no oxygen uptake is detected. If, however, the extract of a boiled washed muscle suspension is added to the test, vigorous oxygen uptake takes place. It is clear that the coenzyme of the $d$-amino acid oxidase, a flavin compound, is present in a bound form, probably as the prosthetic group of a hitherto unknown enzyme.

When cytochrome oxidase is prepared according to the method of Keilin and Hartre ${ }^{2}$, it is found that the flavin nucleotide follows the cytochrome oxidase and the group of insoluble dehydrogenases. I have now found that a flavo-protein can be extracted from such muscle preparations by the action of dilute alcohol and salt solution. Following this extraction, the flavo-protein has been gradually purified to a flavin phosphate content of 0.65 per cent. This new flavo-protein can be regarded as practically pure, 